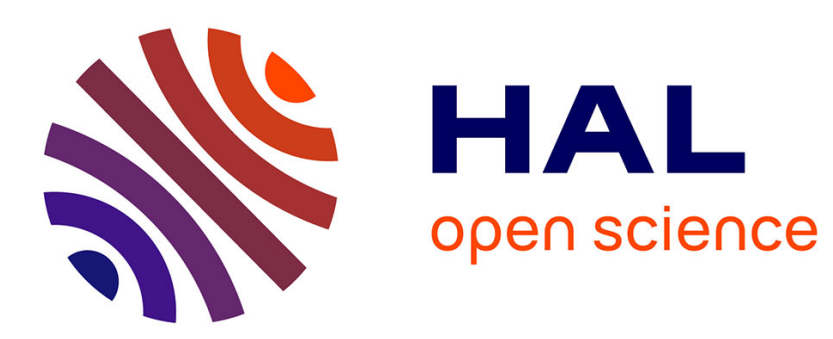

\title{
Performances comparées des machines à aimants et à réluctance variable. Maximisation du couple massique ou volumique \\ D. Matt, Jean-François Llibre
}

\section{- To cite this version:}

D. Matt, Jean-François Llibre. Performances comparées des machines à aimants et à réluctance variable. Maximisation du couple massique ou volumique. Journal de Physique III, 1995, 5 (10), pp.1621-1641. 10.1051/jp3:1995214 . jpa-00249405

\section{HAL Id: jpa-00249405 https://hal.science/jpa-00249405}

Submitted on 1 Jan 1995

HAL is a multi-disciplinary open access archive for the deposit and dissemination of scientific research documents, whether they are published or not. The documents may come from teaching and research institutions in France or abroad, or from public or private research centers.
L'archive ouverte pluridisciplinaire HAL, est destinée au dépôt et à la diffusion de documents scientifiques de niveau recherche, publiés ou non, émanant des établissements d'enseignement et de recherche français ou étrangers, des laboratoires publics ou privés. 
Classification

Physics Abstracts

02.70

\title{
Performances comparées des machines à aimants et à réluctance variable. Maximisation du couple massique ou volumique
}

\author{
D. Matt et J.F. Llibre \\ Laboratoire d'Electrotechnique de Montpellier, Université Montpellier II, Place Eugène Ba- \\ taillon, CC 79, 34095 Montpellier Cedex 5, France
}

(Reçu le 25 avril 1994, révisé le 26 septembre 1994 et le 11 maj 1995, accepté le 17 juillet 1995)

\begin{abstract}
Résumé. - L'objet de cet article est de comparer directement, pour la première fois à notre connaissance, les performances de machines électriques mettant en œuvre trois principes de fonctionnement différents, très récemment exploités. Nous examinons les possibilités de réalisation d'actionneurs à fort couple et puissance massique élevée, pour des applications d'entrainement direct, conçus à partir soit d'une structure synchrone classique utilisant des aimants pour l'inducteur, soit d'une structure à réluctance variable vernier soit, enfin, d'un dispositif combinant l'effet de réluctance variable à l'utilisation d'aimants. Pour permettre une comparaison réellement directe, l'architecture des trois moteurs exploitant les concepts évoqués, ainsi que la forme de leurs principales pièces, seront pratiquement identiques.
\end{abstract}

\begin{abstract}
The subject of this article is to compare the performances of electrical machines using three different principles of functioning which are recently exploited. We consider possibilities to realise actuators with high torque and power per unit of mass for direct drive applications. The three different machines involved in the comparison are: (i) the classical synchronous structure with permanent magnets in the inductor, (ii) the vernier reluctance structure, (iii) the vernier reluctance structure with permanent magnets. To allow a good comparison, the architecture of those three motors has to be identical, particularly the shape of the main elements.
\end{abstract}

\section{Introduction}

Les progrès réalisés ces vingt dernières années dans le domaine des matériaux magnétiques ainsi que dans celui des dispositifs électroniques d'alimentation ont ouvert de nouvelles voies d'investigation dans la conception des machines électriques. Les convertisseurs statiques ont permis l'utilisation des moteurs synchrones dans beaucoup d'applications tandis que la mise au point d'aimants permanents de grande qualité, et de matériaux doux à faibles pertes, a abouti à la conception de machines très performantes.

Sur un plan historique, les recherches ont d'abord porté sur l'étude de machines à réluctance variable utilisant des systèmes de petits plots magnétiques excités par des bobinages globalisés (l'échelle géométrique de bobinage est dissociée de l'échelle de denture). Il s'agissait de montrer 
que, pour ces dispositifs, à condition d'opter pour des saillances de très petite dimension, il est possible d'atteindre des couples massiques importants $[1-3,7]$.

Presque simultanément, sont apparues les premières machines synchrones à aimants. Il est vite apparu que, pour des machines de petite et moyenne taille, il était très avantageux, dans une structure classique, de remplacer le bobinage d'inducteur par des aimants [4]. Le marché de ces machines a crû de façon spectaculaire en quelques années, elles sont maintenant très utilisées en tant que servomoteurs.

Enfin, plus récemment, les premières machines à réluctance variable utilisant des aimants ont été étudiées. Une partie des concepts mis en œuvre pour les dispositifs à réluctance variable pure ont été repris avec bien plus de succès $[6,8,13-18]$.

Nous comparons ici ces trois types de machine. Si les différents principes de fonctionnement ont déjà fait, partiellement, l'objet de comparaisons globales, nous effectuons pour la première fois, à notre connaissance, une comparaison directe des performances de machines fonctionnant dans les mêmes conditions et possédant des structures très similaires. Le cadre d'analyse concerne l'élaboration de dispositifs à performances massiques ou volumiques élevées, pour des applications d'entraînement direct.

\section{Bases de la comparaison}

2.1. GÉNÉRALITÉs. - Le problème majeur pour ce type de comparaison est que les architectures globales des machines utilisant les trois concepts évoqués ont tendance à être très différentes les unes des autres. La machine à aimant classique est traditionnellement cylindrique. Les machines à réluctance variable peuvent avantageusement être discoïdes, homopolaires multistack ou polyphasées à circuit unique $[6-8,10,13,14]$. Il est évident que, dans ces conditions, les géométries de bobinage et les formes des machines, en raison de leur disparités, n'autorisent pas de comparaisons réellement directes.

Fort heureusement, il existe un agencement qui peut être identique pour les trois dispositifs. L'architecture commune sera cylindrique, avec un induit polyphasé encoché. La base est celle de la machine classique. Les machines à réluctance variable avec et sans aimants seront alors à structure vernier $[1,3,7,16,18]$.

\subsection{Cadre de l'Étude. Description de la STRuCture de Base}

2.2.1. Généralités. - L'objet de cette étude est d'établir les possibilités des trois dispositifs lorsque l'on cherche à maximiser le couple pour un poids ou un encombrement doniré. Des vitesses de rotation de l'ordre du millier de tours par minute devront pouvoir être atteintes pour des fréquences de fonctionnement raisonnables (inférieures à $800 \mathrm{~Hz}$ ).

Pour être valable la comparaison doit être effectuée pour des niveaux de pertes Joule et des fréquences de fonctionnement (à vitesse donnée) similaires.

Un certain nombre de calculs de dimensionnement seront assez approximatifs, mais il ne peut en être autrement si l'on veut d'une part, conserver au propos un caractère suffisamment général, d'autre part, ne pas trop alourdir les différents développements. Nous nous sommes attachés toutefois à conserver la meilleure précision possible au niveau des comparaisons de dimensions entre machines. Pour les mêmes raisons, nous limiterons autant que faire se peut le nombre de paramètres (degrés de liberté) intervenant dans les calculs. 

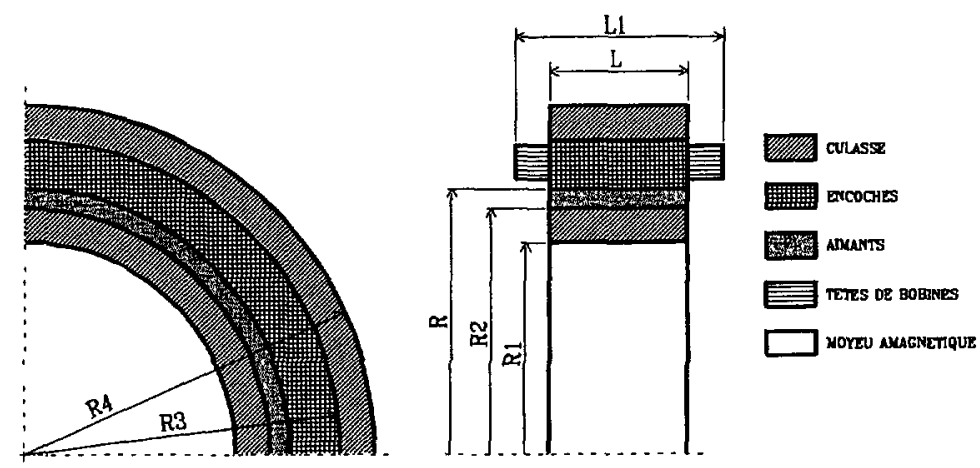

Fig. 1. - Structure de base.

[Basic structure]

2.2.2. Les dimensions de la structure de base. - Les machines étudiées ont globalement la structure indiquée sur la figure $1\left(^{1}\right)$

Les dimensions des différentes zones sont reportées dans le tableau ci-dessous :

\begin{tabular}{|c|c|c|}
\hline Cote & Désignation & Valeur calculée \\
\hline$R$ & rayon à l'entrefer & - \\
$e$ & jeu d'entrefer & - \\
$L$ & Longueur utile (longueur du fer) & - \\
$L_{1}$ & encombrement total en longueur & - \\
$h_{\mathrm{c}}$ & épaisseur des culasses & $h_{\mathrm{c}}=R_{2}-R_{1}=R_{\mathrm{A}}-R_{3}$ \\
$a$ & épaisseur de la couronne d'aimants & $a=R-R_{2}$ \\
$h$ & épaisseur de la zone des encoches & $h=R_{3}-R$ \\
$h_{\mathrm{b}}$ & hauteur des têtes de bobines & $h_{\mathrm{b}}=\left(L_{1}-L\right) / 2$ \\
\hline
\end{tabular}

A partir de ces cotes, nous précisons certaines dimensions :

- volume utile de la machine (sans les têtes de bobine), $V_{u}$. tube d'épaisseur $R_{4}-R_{1}$ et de longueur $L$,

- hauteur des têtes de bobines, $h_{\mathrm{b}}$ : nous la calculons, en première approximation, de la manière suivante :

$$
h_{\mathrm{b}} \cong \frac{\pi R}{4 p}, \text { où } p \text { représente le nombre de paires de pôles, }
$$

- épaisseur de culasse, $h_{c}$. elle est liée à la surface des pôles et au rapport de l'induction radiale $B_{\mathrm{e}}$ dans l'entrefer, sous un pôle, à l'induction admise dans la culasse, $B_{\mathrm{c}}$.

$$
h_{\mathrm{c}}=\frac{\pi R B_{\mathrm{e}}}{2 p B_{\mathrm{c}}}
$$

Pour caractériser la forme de la machine nous introduisons le coefficient d'allongement $k_{\mathrm{f}}=$ $L / 2 . R$.

$\overline{\left({ }^{1}\right) \text { la zone d'entrefer, de faible épaisseur }}(e)$, n'est pas représentée sur la figure. 
2.2.3. Les courants, aspect thermique. - Du point de vue de la création de f.m.m., la zone des encoches est assimilable à une couche de courant uniforme, d'épaisseur $h$. Nous admettrons que la répartition azimutale de la densité de courant $j$ dans la zone homogénéisée est de la forme :

$$
j=j_{0} \cos (p \theta) \text { avec } j_{0}=\sqrt{2} k_{\mathrm{r}} J
$$

où $J$ représente la densité de courant efficace réelle dans les conducteurs d'induit et $k_{\mathrm{r}}$ le taux de remplissage global en cuivre dans la zone des encoches (en tenant compte de la présence du fer).

La couche de courant d'épaisseur $h$ est parfois remplacée par une couche de courant mince équivalente, le produit $J k_{\mathrm{r}} h$ représente alors (si $h$ est petit devant $R$ ) la densité linéique, $\lambda$, du courant parcourant la couche mince. On montre alors que l'échauffement est proportionnel au produit $J \lambda$. Pour une élévation de température de $100^{\circ} \mathrm{C}$, il est d'usage d'adopter :

$$
J \lambda=10^{11} A^{2} / \mathrm{m}^{3}
$$

Toutes nos comparaisons seront faites en utilisant cette valeur.

\section{Spécificités de chacune des trois structures}

3.1. LA MACHINE SYNCHRONE À AIMANTS. - Nous adoptons une structure classique, en tout point conforme au schéma de la figure 1. En particulier, les aimants occuperont toute la surface d'un pôle et remplissent donc uniformément la zone qui leur est affecté.

La recherche de grands couples massiques s'effectue en utilisant un nombre de pôles élevé.

Le couple moteur dépend directement du champ créé par les aimants seuls. Celui-ci a l'allure représentée sur la figure 2 . Une propriété très intéressante de ces machines est que l'induction moyenne, $B_{\mathrm{a}}$, sous un pôle est pratiquement indépendante de la dimension du pôle [4], elle est de la forme:

$$
B_{\mathrm{a}}=B_{\mathrm{r}} \frac{a}{e+a}
$$

où $B_{\mathrm{r}}$ représente l'induction rémanente de l'aimant.

Lorsque le nombre de pôles augmente, la quantité relative de fuites interpolaires augmente (part de flux qui se referme localement entre deux aimants jointifs et qui ne participe pas à la création de couple), mais elle reste insignifiante tant que le rapport de l'entrefer $e$ au pas polaire $\pi R / p$ reste faible [11], ce qui sera toujours le cas.

Lorsque les aimants sont jointifs, la forme exacte de l'onde d'induction est très proche d'un rectangle, nous utiliserons ce modèle (Fig. 2) en prenant:

$$
B_{\mathrm{a}} \cong 0,8 B_{\mathrm{r}}
$$

qui est une valeur approchée dont la précision est suffisante. Ce modèle fait disparaître, à dessein (cf. paragraphe 2.2.1.), deux paramètres de calcul : l'épaisseur des aimants et celle de l'entrefer. Nous en tiendrons compte toutefois dans le dimensionnement en adoptant une épaisseur d'aimant suffisante pour garantir une valeur de champ démagnétisant, dans l'aimant, éloignée de la valeur critique (laquelle valeur est de toute façon extrêmement difficile à atteindre dans une machine classique, pour les nuances d'aimants dont il sera fait usage). Conformément à ce qu'il ressort de la formulation de $B_{\mathrm{a}}$ donnée en début de paragraphe, l'épaisseur d'aimant sera toujours suffisante pour que celle de l'entrefer n'ait pas d'influence significative sur les performances. 


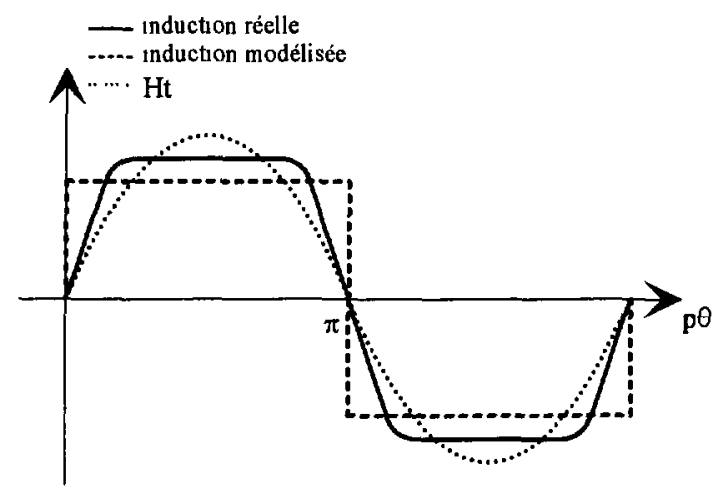

Fig. 2. - Allure de l'induction dans l'entrefer.

[Induction in the air gap versus angular coordinate characteristic.]

Le couple, $\Gamma$, de la machine se calcule alors par :

$$
\Gamma=R^{2} L \int_{0}^{2 \pi} B_{\mathrm{n}} H_{\mathrm{t}} \mathrm{d} \theta=2 p R^{2} L B_{\mathrm{a}} \int_{0}^{\pi / p} H_{\mathrm{t}} \mathrm{d} \theta
$$

la composante tangentielle du champ dans l'entrefer $H_{\mathrm{t}}$ s'exprime à partir de $\lambda$ :

$$
H_{\mathrm{t}}=\sqrt{2} \lambda \sin (p \theta)
$$

d'où :

$$
\Gamma=8 \sqrt{2} k_{\mathrm{f}} B_{\mathrm{a}} \lambda R^{3}, \text { avec } \lambda J=10^{11}
$$

Le seul degré de liberté pour effectuer le dimensionnement de la machine réside dans le choix de $J$, d'où découle la valeur de la cote d'épaisseur $h$ de la zone des encoches.

Pour achever le dimensionnement, il reste à préciser l'encombrement des culasses, soit la valeur de $h_{c}$. Elle est liée au niveau moyen d'induction dans l'entrefer $B_{\mathrm{e}}$ qui, en pratique, n'est que peu affecté par le champ de réaction d'induit, lorsque le nombre de pôles est élevé, d'où : $B_{\mathrm{e}} \cong B_{\mathrm{a}}$. En prenant $B_{\mathrm{c}}=1,4 \mathrm{~T}$ et $B_{\mathrm{r}}=1 \mathrm{~T}$, nous obtenons :

$$
h_{\mathrm{c}} \cong 0,9 \frac{R}{p}
$$

Enfin nous pouvons donner l'ordre de grandeur du facteur de puissance $f_{\mathrm{p}}$ pour un fonctionnement au couple maximal (répartition de champ conforme à la Fig. 2) :

$$
f_{\mathrm{p}}=\cos \left(\arctan \left(b_{\mathrm{r}} / b_{\mathrm{a}}\right)\right)
$$




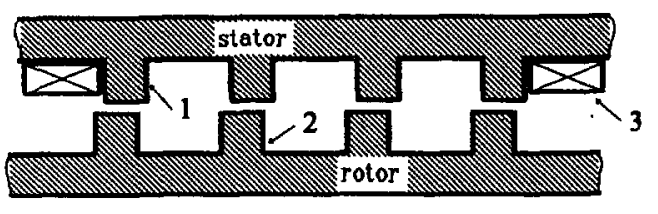

Fig. 3

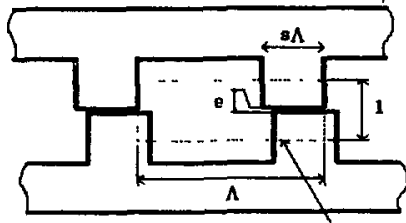

Domaine élémentaire

Fig. 4

Fig. 3. - Schéma de principe d'une machine à réluctance variable.

[Reluctance design.]

Fig. 4. - Domaine élémentaire.

[Elementary domain.]

où $b_{\mathrm{r}}$ représente le champ d'induction de réaction d'induit et $b_{\mathrm{a}}$ le fondamental de l'induction due aux aimants :

$$
b_{\mathrm{r}}=\mu_{0} \frac{\sqrt{2} R \lambda}{a p}, b_{\mathrm{a}}=\frac{4}{p} 0,8 B_{\mathrm{r}}
$$

\subsection{LA MACHINE VERNIER À RÉLUCTANCE VARIABLE}

3.2.1. Généralités sur les machines à réluctance variable. - Les dispositifs qui nous intéressent ici exploitent un mode de fonctionnement qui peut être expliqué à l'aide des schémas de principe donnés sur les figures 3 et 4.

Le couplage a lieu par interaction de systèmes de plots magnétiques, rotoriques (2) et statoriques (1), excités par un bobinage globalisé (3). Le système de plots, soumis au champ d'excitation créé par la bobine tendra à adopter la position de réluctance minimale (conjonction des plots), indépendamment du sens du champ.

La caractéristique principale de ce dispositif est que l'échelle géométrique de bobinage, définie par la répartition de la f.m.m. est complètement dissociée de l'échelle de denture. L'échelle de bobinage est dite globale tandis que celle de denture, locale. Nous verrons plus loin ce que cela implique.

Dans ces structures, la densité surfacique d'effort moteur, associée à la surface active (surface d'entrefer), est entièrement conditionnée par les proportions, et non les cotes dans l'absolu, de la cellule de répétitivité du système de plots. Celle-ci définit le domaine élémentaire d'étude de la machine. Sur la figure 4 nous retrouvons les principales cotes caractérisant un motif de hauteur unitaire.

Pour obtenir des efforts moteurs intéressants, il est nécessaire de saturer les plots. Ceci a pour conséquence d'interdire toute formulation analytique précise du comportement. Les performances intrinsèques des motifs étudiés sont alors obtenues par des calculs du champ à l'aide de logiciels de calcul par éléments finis. Ils sont présentés sous forme de courbes paramétrées par les cotes de proportion.

Pour nos dimensionnement de machines nous utiliserons des résultats déduits de la référence [2]. Les deux paramètres importants sont l'entrefer relatif $e$ et la longueur relative $\Lambda$ (rappelons que le motif a une hauteur unitaire). Il a été montré que la proportion de fer présentait un 


$$
e=0,01
$$

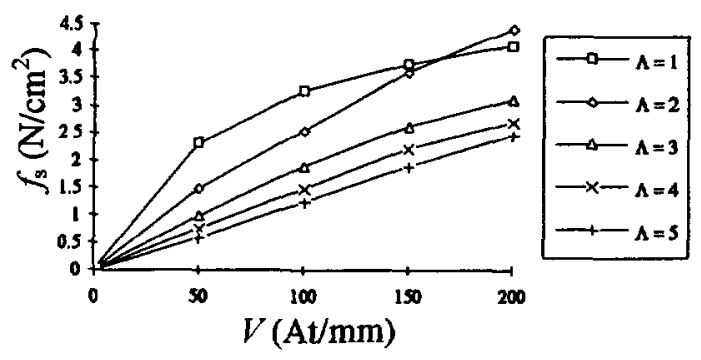

$$
e=0,07
$$

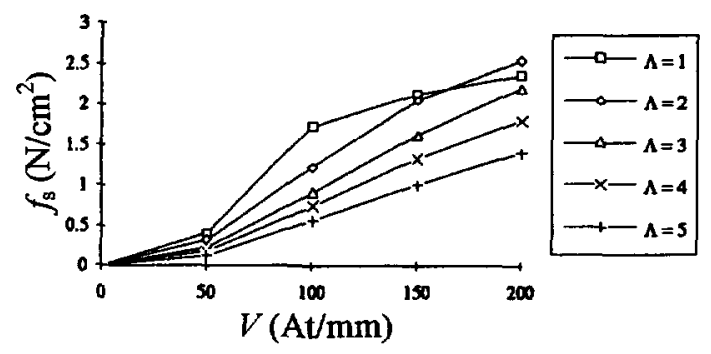

$$
e=0,04
$$
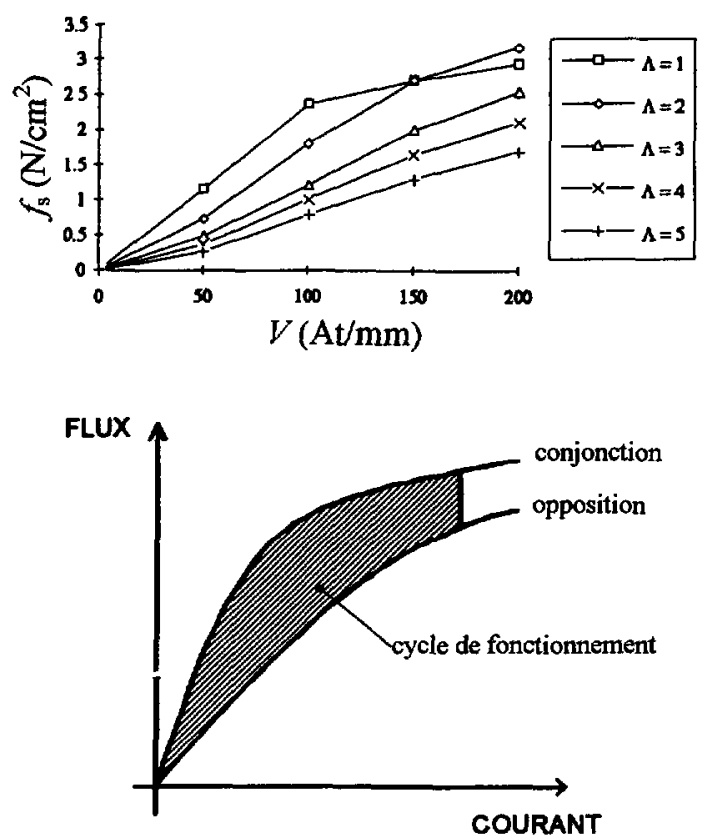

Fig. 5. - Efforts moteurs par unité de surface d'entrefer.

[Stress per unit of air gap area.]

optimum pour $s \cong 5 / 12$. Les courbes de la figure 5 représentent l'évolution de l'effort surfacique $f_{\mathrm{s}}$ en fonction du potentiel scalaire magnétique appliqué aux bornes du domaine, $V$. Les motifs sont alimentés en créneaux de courant ( $V$ représenterait alors l'amplitude du créneau de courant excitant un motif d'un mètre d'épaisseur), le cycle de fonctionnement est conforme à ce qui est représenté.

L'avantage de disposer d'échelles géométriques dissociées est mis en évidence par le raisonnement qui suit. Prenons une machine dont nous connaissons la dimension des motifs à réluctance variable (plots) et la quantité de cuivre nécessaire pour obtenir un effort moteur donné. Supposons maintenant que nous puissions réduire l'ensemble des cotes du système de plots d'un même facteur. Si le courant d'excitation est réduit dans les mêmes proportions nous retrouvons rigoureusement les mêmes champs et par conséquent les mêmes efforts moteurs. Les deux machines, homothétiques l'une de l'autre, présentent absolument les mêmes caractéristiques électriques, à ceci près que la fréquence d'alimentation de la seconde a crû comme l'inverse du rapport d'homothétie.

La conséquence immédiate de l'application de ce principe serait que l'encombrement global des parties "utiles" de la machine tendrait vers celui, uniquement, du circuit de fermeture du flux, pour de grands rapports d'homothétie. Les pertes Joule dans le cuivre tendraient, elles, vers zéro puisque les courants tendent eux mêmes vers zéro. Des performances élevées peuvent alors être obtenues par l'accroissement des fréquences de fonctionnement, sans sacrifice sur le rendement. 


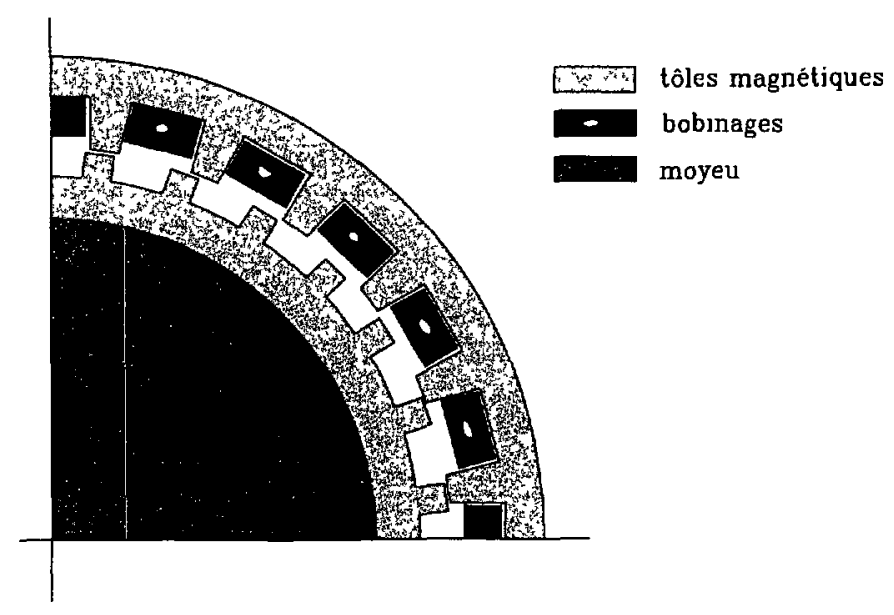

Fig. 6. - Machine vernier à réluctance variable.

[vernier reluctance machine.]

Le principe que nous venons d'évoquer se heurte toutefois à une difficulté majeure de mise en œuvre. Les efforts obtenus dans les dispositifs à réluctance variable sont très dépendants de la valeur de l'entrefer. Ce dernier doit être le plus petit possible compte tenu des contraintes mécaniques de réalisation. L'homothétie concerne alors l'ensemble des cotes du motif sauf l'entrefer qui augmente en valeur relative. L'accroissement de l'entrefer relatif tend à annuler les effets escomptés, néanmoins le bilan reste globalement positif.

3.2.2. La machine vernier. - Sa structure est représentée sur la figure 6 . Nous voyons qu'il convient, pour se ramener à la structure de base (Fig. 1), de remplacer la couche d'aimants par une couche de plots rotoriques.

Nous ne rappelons ici que très succinctement le principe de fonctionnement de cette machine qui a déjà fait l'objet de descriptions détaillées $[1,3,7]$. L'excitation des plots est obtenue à l'aide d'un bobinage à champ tournant, logé dans des encoches, exactement similaire à celui d'un induit de machine classique. Les encoches ouvertes réalisent le système de plots statoriques. Le nombre de dents rotoriques est différent du nombre de dents statoriques, si bien que la perméabilité locale au niveau de l'entrefer est fonction de la coordonnée azimutale. Au cours du fonctionnement, ce sont les zones de réluctance minimale qui vont tourner en synchronisme avec le champ, le rotor tournant, lui, à une vitesse inférieure.

La condition de bon fonctionnement est que l'onde de perméabilité d'entrefer ait une périodicité double de celle de f.m.m. pour une machine non excité. Le pas angulaire, $\Theta$, de répartition de perméabilité valant :

$$
\Theta=\frac{2 \pi}{\left|N_{\mathrm{r}}-N_{\mathrm{s}}\right|}
$$

où $N_{\mathrm{r}}$ et $N_{\mathrm{s}}$ représentent respectivement le nombre de dents rotoriques et statoriques. Nous obtenons donc:

$$
\left|N_{\mathbf{r}}-N_{\mathrm{s}}\right|=2 p
$$




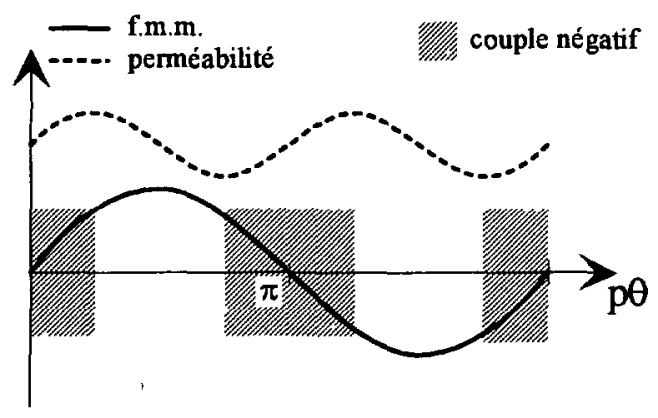

Fig. 7. - Couplages négatifs dans les machines vernier.

[Negative torque area in vernier reluctance machines.]

La pulsation de rotation du rotor, $\Omega_{\mathrm{r}}$, est alors liée à la pulsation des courants d'induit, $\omega$, par :

$$
\Omega_{\mathrm{r}}=\frac{2 \omega}{N_{\mathrm{r}}}
$$

Ce mode de réalisation de machine à réluctance variable utilise les principes énoncés au paragraphe 3.2.1. Les efforts moteurs peuvent être déduits des courbes paramétrées, toutefois les plots ne sont pas excités de façon tout à fait identique à ce qui est représenté sur le cycle de la figure 5. Deux phénomènes tendent à diminuer le couplage. Sur la figure 7, nous représentons l'allure de la f.m.m. et de la perméabilité d'entrefer. Nous voyons que la première différence avec le cycle "théorique" est que le potentiel appliqué aux bornes des motifs n'est plus carré, mais sinusoïdal. La seconde différence réside dans le fait que la totalité de la surface d'entrefer n'est pas productrice d'effort moteur. En effet, quelque soit la répartition des courants, il existera toujours des zones introduisant des couples "négatifs". Ce phénomène est longuement expliqué dans la référence [1]. Il n'est pas possible de chiffrer dans l'absolu la dégradation de l'effort surfacique, elle dépend de beaucoup de paramètres et les fonctions ne sont pas linéaires. Toutefois, l'expérience montre que, d'une manière générale, un affaiblissement de $30 \%$ est une bonne valeur approchée.

Le couple moteur, $\Gamma$, peut alors être formulé de la manière suivante :

$$
\Gamma=0,7 f_{\mathrm{s}} k_{\mathrm{f}} 4 \pi R^{3}
$$

Le potentiel réduit d'excitation, $V$, (f.m.m. ramenée à la hauteur du motif élémentaire), duquel nous déduisons la valeur de l'effort surfacique, $f_{\mathrm{s}}$, à l'aide des courbes de la figure 5 , vaut :

$$
V=\frac{\sqrt{2} \lambda \mathrm{R}}{l p}
$$

où $l$ représente la hauteur réelle des motifs de plots. Cette relation permet de mettre en évidence une caractéristique des dispositifs à petits plots et bobinages globalisés. Le potentiel d'excitation étant proportionnel à l'échelle globale, il n'est pas nécessairement judicieux d'adopter, dans ces machines, un nombre élevé de pôles, comme dans les machines classiques, pour la 

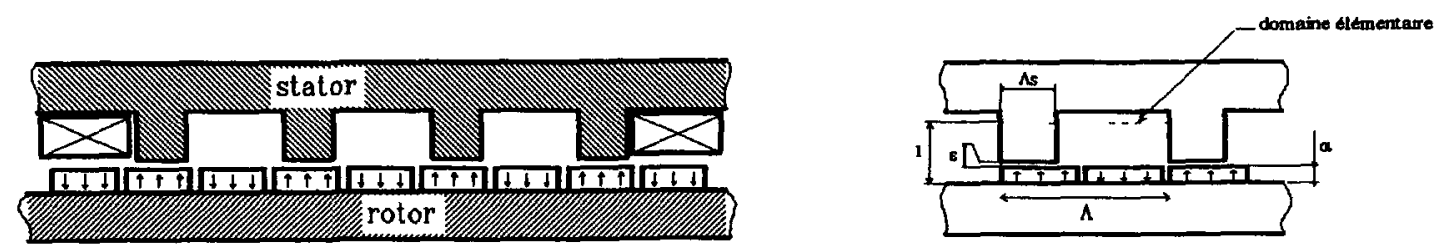

Fig. 8. - Schéma de principe et domaine élémentaire.

[Reluctance magnet design and elementary domain.]

recherche de performances massiques élevées. Le nombre de pôles n'est ici qu'un paramètre supplémentaire de recherche d'optimum. Il s'ajoute aux paramètres suivants : $V$ (ou $J$ ) et $\Lambda$.

De la même façon que pour la machine précédente, nous pouvons préciser l'encombrement des culasses. La répartition d'induction est à peu près sinusoïdale. Les motifs étant systématiquement saturés pour des raisons déjà évoquées, nous admettrons que la valeur maximale est égale à $s B_{\mathrm{s}}$, soit environ $0,7 \mathrm{~T}\left(s=5 / 12, B_{\mathrm{s}}=1,6 \mathrm{~T}\right)$. D'où :

$$
h_{\mathrm{c}} \cong 0,5 \frac{R}{P}
$$

\subsection{LA MACHINE VERNIER À AIMANTS}

3.3.1. Généralités sur les machines à aimants et réluctance variable. - Ces dispositifs représentent une évolution des précédents. Schématiquement, il suffit de remplacer la rangée de plots rotoriques par une rangée d'aimants alternés. Nous obtenons alors le schéma de la figure 8 , dérivé de celui de la figure 3 .

La production d'effort moteur dans cette configuration a aussi lieu par effet de réluctance variable, mais c'est la réluctance de l'élément de circuit magnétique associé à un aimant donné qui varie et non la réluctance globale du système de plots. Soumis au champ d'excitation, ce sont les aimants orientés dans le sens du champ qui auront tendance à se positionner en face des plots magnétiques.

Le mode de fonctionnement est assez similaire au précédent. En particulier, il s'agit aussi de dispositifs à échelle globale et locale découplée. Les principes déjà énoncés, pour la recherche de performances élevées, sont alors utilisés exactement de la même façon. La diminution de la taille des plots et donc l'augmentation de la fréquence de fonctionnement s'accompagne d'une diminution du volume du cuivre, pour un nombre de pôles de bobinage donné.

Nous rappelons brièvement les principaux avantages et spécificités des structures à aimants par rapport à celles à réluctance variable pure. D'autres précisions, qui ne sont toutefois pas nécessaires pour la bonne compréhension de ce qui suit, peuvent être obtenues en consultant la bibliographie $[6,8,13,15,17]$. La différence essentielle, pour l'application des concepts qui nous intéressent, réside dans le fait qu'ici la dépendance des efforts moteurs vis à vis de l'entrefer est très faible. Cela provient du fait que le champ créé par les aimants seuls ne dépend que peu de l'entrefer, tant que ce dernier conserve une dimension faible devant l'épaisseur d'aimant. Un autre avantage est lié au cycle de fonctionnement. Celui-ci s'inscrivant dans les quatre quadrants du plan flux-courant, les niveaux de champ et de courant mis en ouvre sont alors plus faibles à effort moteur donné que dans les structures non polarisées. Enfin, les formes d'onde des grandeurs électriques et le facteur de puissance sont meilleurs. 


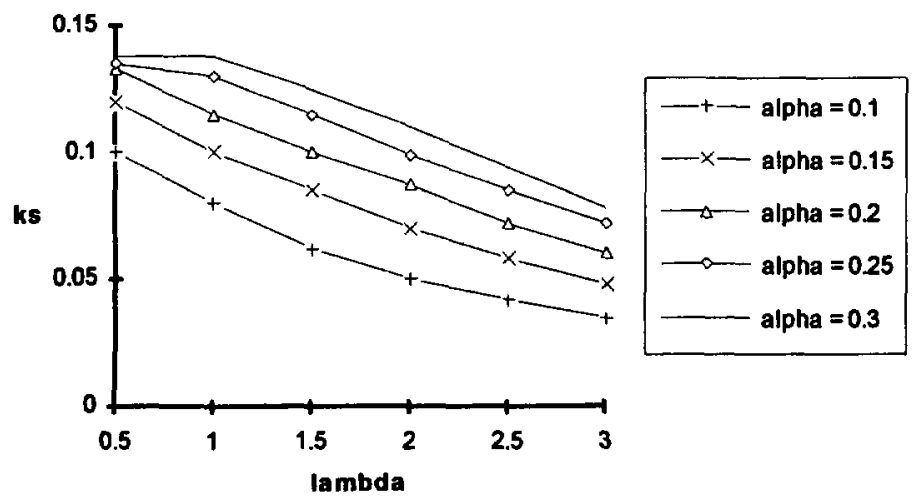

Fig. 9. - Coefficient de couplage.

[Coupling factor.]

Bien que fonctionnant en régime linéaire, la détermination de la densité surfacique d'effort moteur $f_{\mathrm{s}}$ pour les motifs à aimants, ne peut être effectuée avec précision qu'en utilisant le calcul de champ par éléments finis. Nous utiliserons les résultats récents publiés dans les articles $[8,15]$. Il a ainsi été montré que $f_{\mathrm{s}}$ pouvait se mettre sous la forme :

$$
f_{\mathrm{s}}=B_{\mathrm{r}} \frac{a}{e+a} H k_{s}
$$

où $k_{s}$ est un coefficient de couplage dépendant de la forme d'onde du courant d'alimentation et des paramètres de forme du domaine, soit essentiellement $\alpha$ (épaisseur relative d'aimant) et $\Lambda$ (longueur relative du domaine). Nous donnons, sous forme de courbe paramétrées (Fig. 9) la valeur de $k_{s}$ correspondant à une alimentation en régime sinusoïdal au couple maximal (f.e.m. en phase avec le courant). $H$ représente la valeur crête du potentiel magnétique d'excitation du domaine, ramené à la hauteur de l'aimant.

La formulation de $f_{\mathrm{s}}$ fait en outre apparaître le terme classique $a /(e+a)$ qui représente l'influence de l'entrefer sur le couplage. Ici, ce terme est choisi constant et égal à 0,9 (avec des butées sur les épaisseurs d'aimant).

3.3.2. La machine vernier. - Cette structure, représentée sur la figure 10, s'apparente à celle de la figure 6, la rangée de plots rotoriques ayant été remplacée par une couche d'aimants alternés. La constitution du dispositif est alors entièrement conforme au modèle de base (Fig. 1).

Le nombre de paires d'aimants alternés $N_{\mathrm{r}}$ est différent du nombre de dents $N_{\mathbf{s}}$. La condition de bon fonctionnement est alors :

$$
\left|N_{\mathrm{r}}-N_{\mathrm{s}}\right|=p
$$

ce qui conduit à :

$$
\Omega_{\mathrm{r}}=\frac{\omega}{N_{\mathrm{r}}}
$$




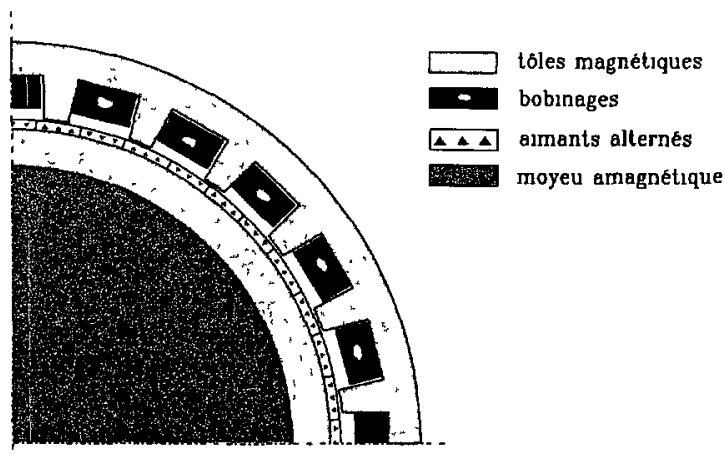

Fig. 10. - Machine vernier à aimants.

[Vernier reluctance machine with permanent magnets.]

Les conditions d'excitation des plots sont les mêmes que celles qui ont servi au tracé du réseau de la figure 9 , le couple moteur s'exprimant alors de la façon suivante $\left({ }^{2}\right)$ :

$$
\Gamma=0,9 \times 4 \pi H k_{s} k_{\mathrm{f}} B_{\mathrm{r}} R^{3}
$$

avec :

$$
H=\frac{\sqrt{2} \lambda R}{a p}
$$

Les paramètres de l'optimisation sont ici : $H$ (ou $J$ ), $\alpha, \Lambda, \mathrm{p}$.

Contrairement à ce que l'on observe dans une machine à aimants classique, le module du champ d'induction de réaction d'induit $b_{\mathrm{r}}$ n'est pas négligeable devant celui créé par les aimants seuls, $b_{\mathrm{a}}$. Ce dernier peut être déduit de l'expression de $f_{\mathrm{s}}[8]$ :

$$
b_{\mathbf{a}}=\frac{\Lambda}{\varepsilon+\alpha} \frac{B_{\mathrm{r}}}{\pi} \frac{\alpha}{\varepsilon+\alpha} k_{s}
$$

au couple maximal, l'amplitude résultante $b$ vaut alors :

$$
b=\sqrt{b_{\mathrm{a}}^{2}+b_{\mathrm{r}}^{2}}, \text { avec } b_{\mathrm{r}}=\mu_{0} H
$$

ce qui nous permet d'estimer l'épaisseur de culasse (cf. paragraphe 2.2.2) :

$$
h_{\mathrm{c}}=\frac{\pi R B_{\mathrm{e}}}{2 p B_{\mathrm{c}}}=\frac{R b}{1,4 p}
$$

Nous pouvons enfin aisément calculer le facteur de puissance :

$$
f_{\mathrm{p}}=\cos \left(\arctan \left(b_{\mathrm{r}} / b_{\mathrm{a}}\right)\right)
$$

( $\left.{ }^{2}\right)$ l'excitation réelle des plots n'est pas purement sinusoïdale, il existe un couplage sur les harmoniques de même rang du champ d'excitation et du champ produit par les aimants, si bien que la formulation du couple donnée précédemment, calculée à partir des seuls fondamentaux, est plutôt pessimiste. Des études en cours montrent que ce phénomène est assez important lorsque le nombre de pôles est grand. 


\section{Comparaison des trois structures}

Nous rappelons que l'objet de cette étude est de présenter les caractéristiques essentielles de trois structures d'actionneur pour des applications d'entraînement direct à fort couple. Les vitesses de rotations envisagées sont basses (inférieures à $1000 \mathrm{t} / \mathrm{mn}$ ), la masse ou l'encombrement de l'actionneur doivent être le plus faible possible.

4.1. Couple massique. - Nous recherchons dans un premier temps un optimum correspondant au couple massique $C_{\mathrm{M}}$ maximal. Le tableau ci-dessous rappelle les degrés de liberté pour l'optimisation, en fonction des structures.

\begin{tabular}{|c|c|c|c|}
\hline Machine & $\mathrm{N}^{\circ}$ & Variables d'optimisation & Principales butées \\
\hline à aimants, classique & 1 & $J$ & $a>1 \mathrm{~mm}$ \\
à r.v, sans aimants & 2 & $p, V$ ou J, & $e>0,05 \mathrm{~mm}$ \\
à r.v, avec aumants & 3 & $p, H$ ou $J, \Lambda, \alpha$ & $a>1 \mathrm{~mm}, e>0,1 \mathrm{~mm}$ \\
\hline
\end{tabular}

La butée d'entrefer a été choisie très faible pour les machines à réluctance variable afin que celles-ci ne soient pas trop désavantagées dans la comparaison, leurs performances dépendant intimement de la valeur de l'entrefer.

Les résultats présentés sur la figure 11 représentent l'évolution de $C_{\mathrm{M}}$ en fonction du rapport $\omega / \Omega_{r}$ pour différentes valeurs du couple nominal $C_{N}$ et du coefficient de forme $k_{\mathrm{f}}$. Les bases du calcul sont celles établies au paragraphe 3 . Le couple nominal est défini par l'échauffement toléré et se rapporte donc au produit $\lambda J$ qui est pris égal à $10^{11}$ pour l'ensemble des structures. Le couple massique est calculé à partir de la masse $M$ des seules pièces utiles, à savoir la zone de volume $V_{\mathrm{u}}$ (cf paragraphe 2.2.2.) ainsi que les têtes de bobinage :

$$
C_{\mathrm{M}}=\frac{C_{\mathrm{N}}}{M}
$$

Les aimants utilisés pour le calcul ont une induction rémanente de $1 T$. Le nombre de phases est fixé à deux ou trois, ce paramètre n'a toutefois pas grande importance pour ces calculs de dimensionnement et n'intervient pas directement.

Cette première étude nous montre que :

- aux basses fréquences, la machine 1 est plus performante que les deux autres, pour des fréquences de fonctionnement un peu plus élevées, les machines 1 et 3 ont des performances similaires tandis que la machine 2 est 30 à $40 \%$ moins performante, nous retrouvons entre autre ici la dégradation des performances due à une mauvaise utilisation des motifs à réluctance variable dans les machines vernier (cf paragraphe 3.2.2),

- lorsque la fréquence croît, la machine 3 devient meilleure que les deux autres, ce qui était prévu, mais nous voyons que ce phénomène est d'autant plus marqué que la machine est de forme allongé $\left(k_{\mathrm{f}}=2\right)$, ceci est dî aux épaisseurs de culasse et aux têtes de bobinages qui sont assez grandes dans les machines à aimants alternés (l'optimisation conduisant à l'utilisation d'un nombre de pôles faible : cf Fig. 12), sauf lorsque le nombre de dents est élevé, et qui interviennent pour beaucoup dans le poids des machines courtes $\left(k_{f}=0,2\right)$,

- dans la recherche de très grands couple massiques, les machines 2 et 3 permettent d'aller plus loin que la machine 1 qui devient vite irréalisable $\left({ }^{3}\right)$ lorsque le nombre de pôles est élevé.

$\left({ }^{3}\right)$ Nous n'avons pas fait apparaitre la limite de faisabilité de la machine 1 , elle est liée aux possibilités de réalisation d'un encochage de petite dimension. Les caractéristiques concernant cette machine ont été prolongées pour montrer la tendance, mais seul le tout début des courbes correspond à une machine réalisable en pratique. 
$C=10 \mathrm{mN}, \mathrm{kf}=0.2$

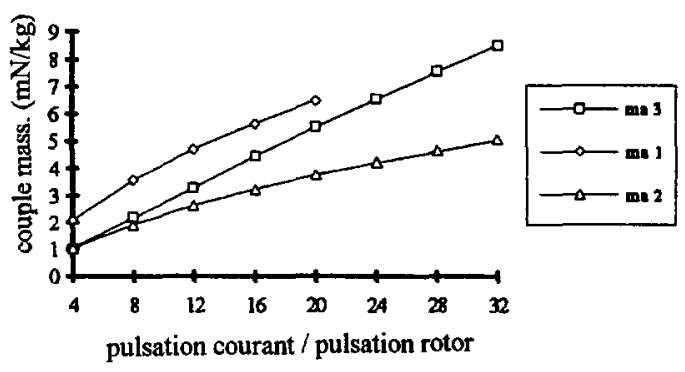

$\mathrm{C}=100 \mathrm{mN}, \mathrm{kf}=0.2$

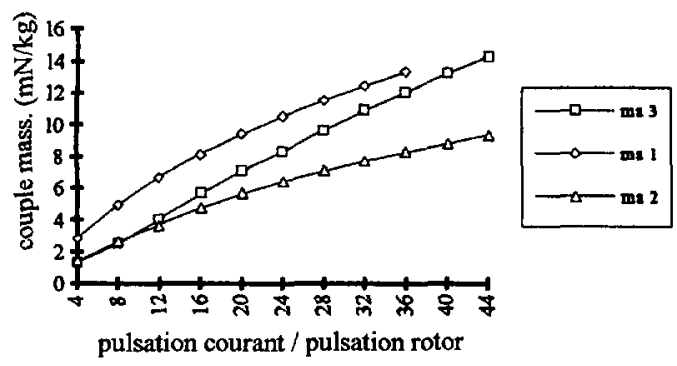

$C=10 \mathrm{mN}, \mathrm{kf}=2$

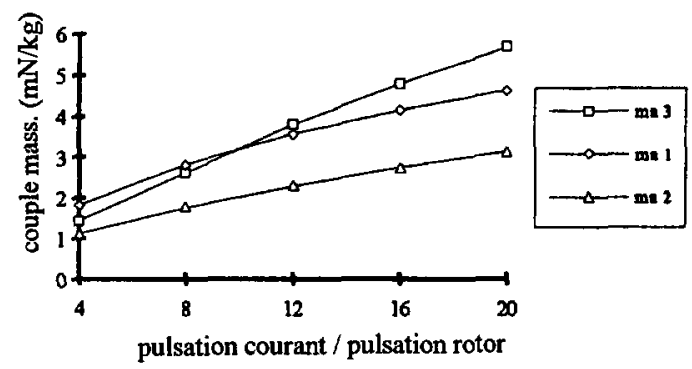

$\mathrm{C}=100 \mathrm{mN}, \mathrm{kf}=2$

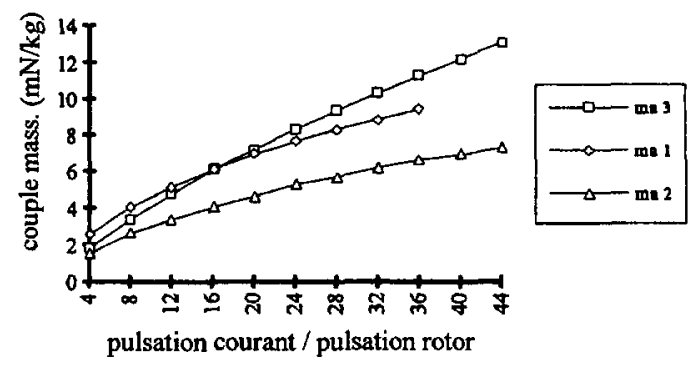

Fig. 11. - Couple massique.

[Torque per unit of mass.]

Un couple massique de l'ordre de $5 \mathrm{mN} / \mathrm{kg}$ est envisageable pour la machine 1 (couple de $100 \mathrm{mN}$ ), tandis que la machine 3 permettrait d'obtenir environ $15 \mathrm{mN} / \mathrm{kg}$. Rappelons que ces chiffres ne prennent pas en compte la totalité du poids des moteurs. Pour les petits moteurs (couple inférieur à $10 \mathrm{mN}$ ), seule la machine 3 est performante si l'on ne s'intéresse qu'au couple massique.

Nous précisons sur la figure 12 l'évolution du nombre de pôles pour la machine 3 , lorsque le couple vaut $100 \mathrm{mN}$. Dans la formulation du couple donnée au paragraphe 3.3.2, le nombre de pôles intervient au dénominateur, il est donc normal d'obtenir, dans l'optimisation, des valeurs relativement faibles pour ce nombre.

Lorsque les aimants mis en cuvre ont une aimantation plus faible $\left(B_{\mathrm{r}}=0,5 \mathrm{~T}\right)$, nous constatons (Fig. 13) que l'allure générale des caractéristiques est inchangée mais que la machine 3 n'est vraiment intéressante que pour des fréquences de fonctionnement élevées, ce qui limite un peu son intérêt dans ce cas de figure.

Nous pouvons justifier ce phénomène par le fait que, conformément à ce que nous venons d'expliquer concernant l'évolution du nombre de pôles, des efforts moteurs conséquents ne peuvent être obtenus qu'avec des valeurs du champ $H$ élevées, qui imposent d'utiliser un nombre de pôles restreint et donc des culasses relativement épaisses. 


\section{$\mathrm{C}=100 \mathrm{mN}$}

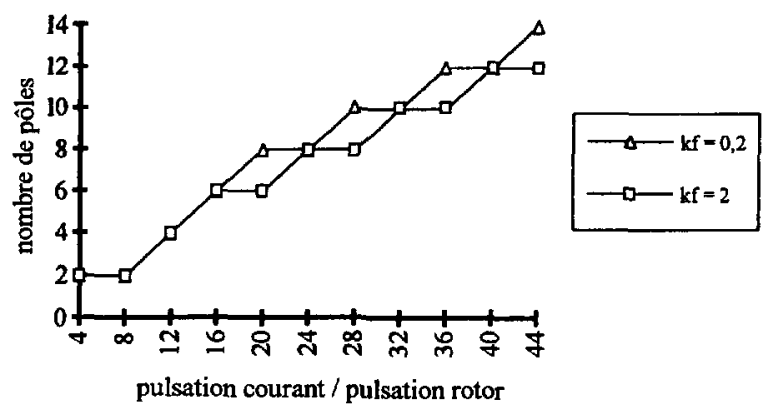

Fig. 12. - Nombre de pôles.

[Number of poles.]

$C=100 \mathrm{mN}, \mathrm{kf}=2, \mathrm{M}=0.5$

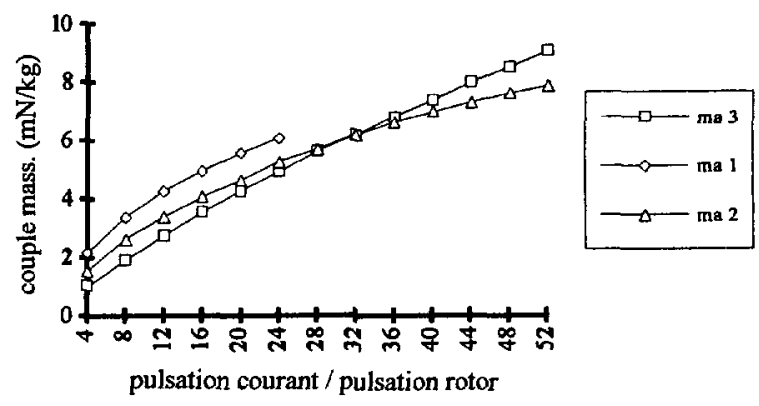

Fig. 13. - Couple massique, aimantation à $0,5 \mathrm{~T}$.

[Torque per unit of mass, magnetization of $0.5 \mathrm{~T}$.]

4.2. Couple volumique. - L'optimisation porte maintenant sur le couple volumique, $C_{V}$ :

$$
C_{V}=\frac{C_{\mathrm{N}}}{V_{\mathrm{T}}}
$$

où $V_{\mathrm{T}}$ est représentatif, cette fois, de l'encombrement global de la machine :

$$
V_{\mathrm{T}}=\pi R_{4}^{2} \mathrm{~L}
$$

Si le volume disponible pour loger le moteur est restreint, il n'est pas forcément possible d'obtenir le couple souhaité, même si l'on fait abstraction du poids ou du rendement.

Nous ne tenons pas compte, ici, de l'encombrement des têtes de bobinage. Ces dernières peuvent s'intégrer de différente façon dans la structure de la machine, il est par conséquent difficile de chiffrer avec une bonne précision leur influence. Nous limiterons, de plus, nos comparaisons aux machines longues, dont les têtes sont courtes en valeur relative. 


\section{$C=100 \mathrm{mN}, k f=2, M=1$}
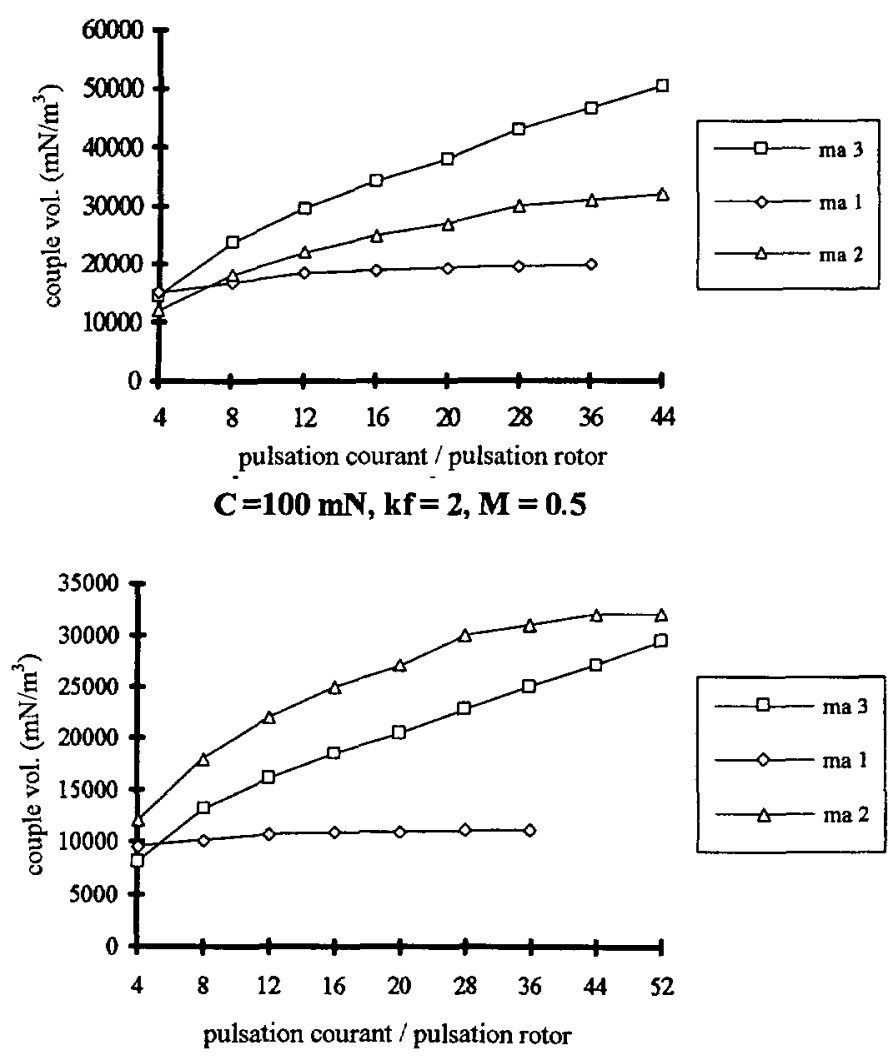

Fig. 14. - Couple volumique.

[Torque per unit of volume.]

Les résultats obtenus sont très significatifs (Fig. 14). Ils sont liés directement aux possibilités d'obtenir des efforts surfaciques élevés. Nous voyons ainsi que, pour la machine classique, la quantité de cuivre nécessaire à l'obtention d'un effort moteur donné étant sensiblement indépendante du nombre de pôles, il n'est pas possible d'accroître les efforts, à échauffement donné, en augmentant la fréquence de fonctionnement. Les machines à réluctance variable permettant, elle, de le faire. Pour des aimants aimantés à $1 \mathrm{~T}$, la machine 3 est, de loin, la plus performante, il est possible d'obtenir des encombrements trois fois plus faibles qu'avec la machine 1.

Un calcul d'ordre de grandeur de l'effort surfacique $f_{\mathrm{s}}$ permet de retrouver ces résultats. Pour la machine $1, f_{\mathrm{s}}=\sqrt{2} \lambda \cdot(2 / \pi) B_{\mathrm{a}}$ (cf. paragraphe 3.1). Avec une valeur typique de $\lambda$ de $20 \mathrm{kA} / \mathrm{m}\left(J=5 \mathrm{~A} / \mathrm{mm}^{2}\right.$ et $\left.\lambda J=10^{11}\right)$, qu'il n'est guère possible de dépasser notablement en conservant un volume de cuivre raisonnable et en évitant des phénomènes de saturation locale, l'effort surfacique est de $1,8 \mathrm{~N} / \mathrm{cm}^{2}$. Pour la machine $3, f_{\mathrm{s}}=0,9, B_{\mathrm{r}} H k_{\mathrm{s}}$ (cf. paragraphe 3.3.1). Avec un champ $H$ de $500 \mathrm{kA} / \mathrm{m}$ que nous pouvons obtenir (avec une bonne marge quant à la désaimantation) si les aimants sont fins, et une valeur typique de $k_{s}$ de 0,1 , l'effort est alors de 


\section{$C=100 \mathrm{mN}, \mathrm{kf}^{\prime}=2$, couple vol. maximal}

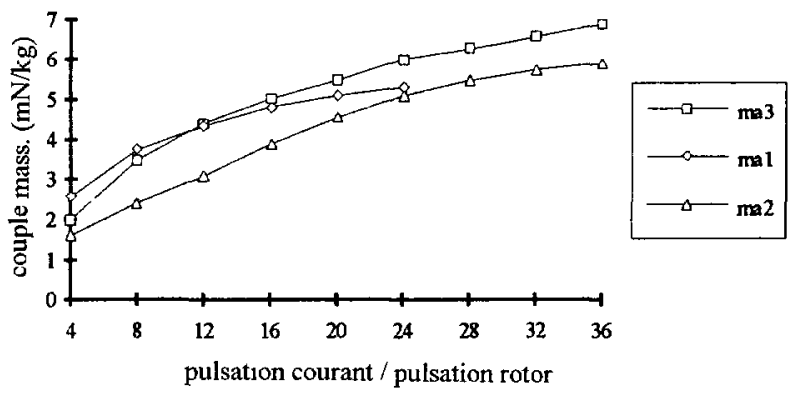

Fig. 15. - Couple massique, machine optimisée au couple volumique.

[Torque per unit of mass (optimization of the torque per unit of volume).]

$4,5 \mathrm{~N} / \mathrm{cm}^{2}$ (il s'agit bien entendu d'une valeur extrême rarement recherchée).

Le tableau suivant résume, à titre d'exemple, les caractéristiques principales de trois machines dimensionnées au couple volumique maximal, avec $\omega / \Omega_{\mathrm{r}}=16$, et $k_{\mathrm{f}}=2$.

\begin{tabular}{|c|c|c|c|}
\hline Caractéristique/machine & ma. 1 & ma. 2 & ma. 3 \\
\hline$C_{\mathrm{v}}\left(\mathrm{mN} / \mathrm{m}^{3}\right)$ & 19000 & 25000 & 34000 \\
masse $(\mathrm{kg})$ & 21 & 30,3 & 21,3 \\
nombre de pôles & 32 & 2 & 2 \\
$R(\mathrm{~mm})$ & 61,7 & 50,6 & 45,2 \\
$J(\mathrm{~A} / \mathrm{mm} 2)$ & 4,5 & 7,6 & 5,4 \\
$h(\mathrm{~mm})$ & 17,5 & 5 & 4 \\
$a(\mathrm{~mm})$ & 1 & - & 3,1 \\
\hline
\end{tabular}

Nous voyons immédiatement que la quantité de cuivre (épaisseur $h$ de la zone des encoches), et donc de courant, est beaucoup plus faible dans le cas des machines verniers que dans celui de la structure conventionnelle. Malgré ceci, l'effort moteur produit, ramené à la surface d'entrefer. vaut $1,7 \mathrm{~N} / \mathrm{cm}^{2}$ pour la machine 1 et $4,3 \mathrm{~N} / \mathrm{cm}^{2}$ pour la machine 3 .

La machine vernier sans aimants s'avère, elle aussi, performante sur le plan des efforts et du couple volumique, elle arrive en première position dans le comparatif avec $B_{\mathrm{r}}=0,5 \mathrm{~T}$. Cette machine, quoique un peu délicate à fabriquer (les performances ne peuvent être obtenues qu'avec des jeux d'entrefer faibles) est une alternative intéressante dans des applications où le coût des aimants peut s'avérer prohibitif.

La figure 15 représente l'évolution du couple massique pour une machine dimensionnée à couple volumique maximal. La dégradation du couple massique dans ces conditions est globalement d'environ $40 \%$.

4.3. Rendement et FaCteur de puissance. - Le facteur de puissance est, d'une manière générale, un point faible des machines à réluctance variable, surtout dans le cadre d'optimisation choisi ici. L'introduction d'aimants dans les motifs à réluctance variable permet d'augmen- 


\section{$C=100 \mathrm{mN}, k f=2$}
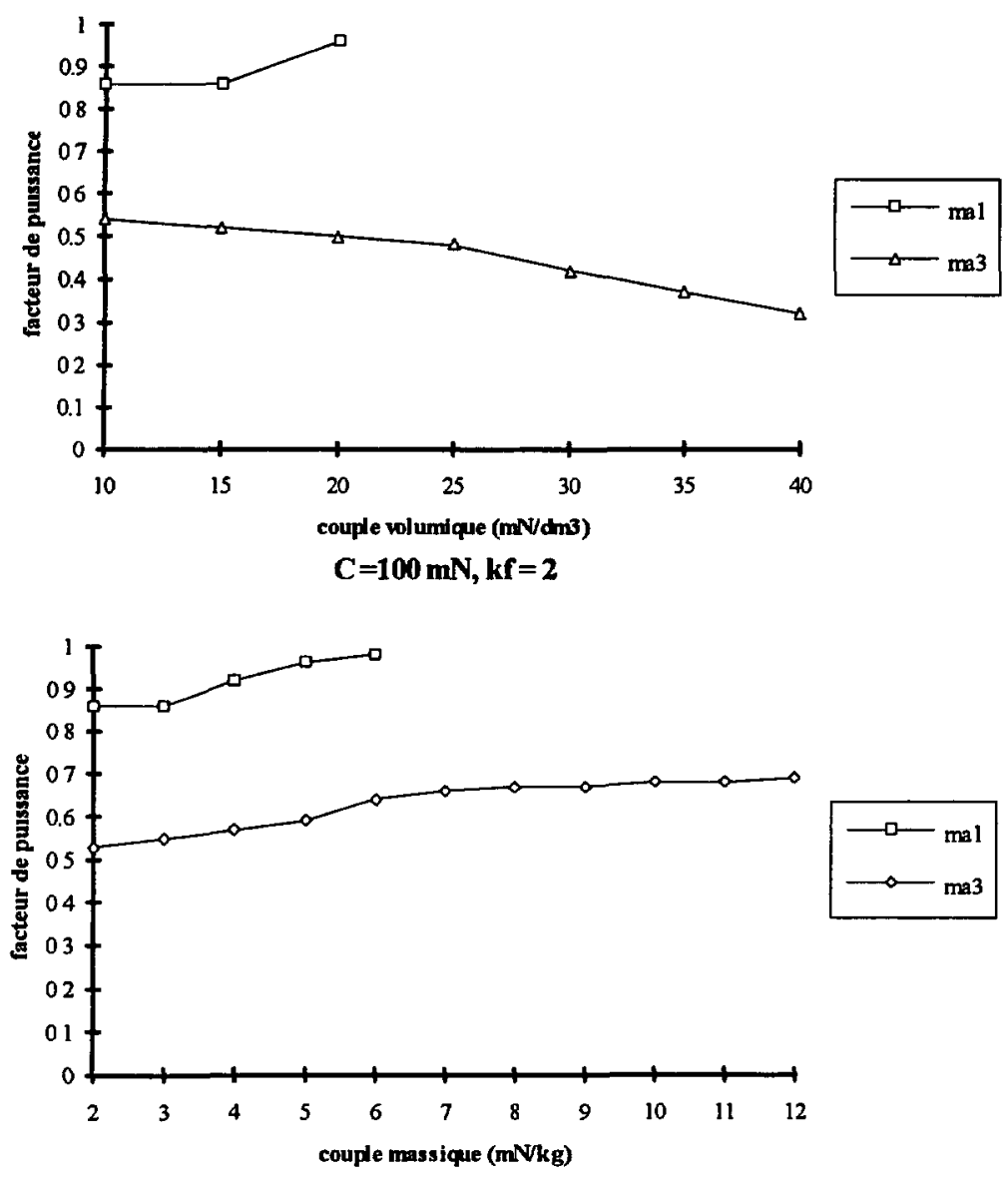

Fig. 16. - Facteur de puissance.

[Power factor.]

ter l'entrefer équivalent vu par les enroulements et tend donc à diminuer le champ de réaction d'induit, et par conséquent à accroître le facteur de puissance. Ce dernier demeure néanmoins modeste comme en atteste la figure 16. Nous ne représentons que les courbes relatives aux machines à aimants (machines 1 et 3 ), dans la mesure où il n'est pas possible de calculer analytiquement, avec une précision suffisante, le facteur de puissance d'une machine vernier à réluctance variable pure.

Pour l'optimisation en couple massique, le facteur de puissance de la machine 3 est compris entre 0,55 et 0,65 et varie peu, tandis que celui de la machine 1 tend vers 1 , ce qui est normal puisque le champ de réaction d'induit est inversement proportionnel aux nombres de pôles (cf. paragraphe 3.1 ). 
Bien entendu, pour l'optimisation en couple volumique, les très bonnes performances de la machine 3 sont obtenues au détriment du facteur de puissance qui est alors compris entre 0,3 et 0,4 . Il s'agit du prix à payer, côté électronique d'alimentation, pour pouvoir bénéficier de performances qu'aucune autre structure ne peut offrir à ce jour.

Nous pouvons donner une estimation des résultats obtenus avec la machine à réluctance variable pure. En effet, de nombreuses études menées dans les années 1980 permettent de s'apercevoir que, sur les machines non excités (dont la structure est la plus proche des dispositifs que nous comparons ici), les facteurs de puissance sont désastreux. Cette grandeur, dans une approche d'optimisation de la puissance volumique, peut avoisiner 0,1 .

Le rendement, calculé à partir des seules pertes Joule, est forcément très bon pour l'ensemble des machines, compte tenu du critère choisi pour le dimensionnement du cuivre (cf. paragraphe 2.2.3). Il est de l'ordre de $97 \%$ à $1000 \mathrm{t} / \mathrm{mn}$ pour une machine de $100 \mathrm{mN}$. Au-delà de ce chiffre peu significatif, il faut arriver à une estimation de la totalité des pertes, et donc de celles dans les matériaux magnétiques. Les deux machines à aimant sont comparables sur ce plan. Dans la machine vernier à aimants le niveau d'induction dans l'entrefer tend à être un peu plus faible que dans la structure classique, les pertes dans les dents sont alors assez faibles mais elles sont compensées par des pertes dans les aimants, qui sont soumis à un champ alternatif. Dans la machine vernier sans aimants, les dents fonctionnent en régime de saturation et sont donc le siège de pertes élevées. Les performances de ces machines sont très sensibles à la qualité des tôles utilisées. Dans tous les cas, le poids du fer étant faible, les pertes dans les circuits magnétiques ont plus d'incidence sur le comportement de la machine (il n'est pas forcément possible de conserver un couple constant lorsque la fréquence d'alimentation augmente) que sur un accroissement significatif des pertes.

4.4. Autres Caractéristiques. - Afin que la comparaison soit la plus complète possible nous donnons, très qualitativement, d'autres caractéristiques de ces moteurs.

La première est un critère économique immédiatement évoqué dès lors qu'il s'agit de moteurs à aimants : la masse des aimants. S'il s'agit d'un frein important à l'heure actuelle $\left({ }^{4}\right)$ pour certaines applications (véhicules électriques ?), d'autres applications (servomoteurs . ) ont eu un développement considérable malgré le coût des aimants. Pour les deux machines à aimants, la quantité d'aimant à couple donné, décroît (en première approximation) lorsque les performances massiques (ou volumique) croissent. La structure vernier est un peu meilleure sur ce plan que la structure classique. Il faut environ $1 \mathrm{~kg}$ d'aimant pour cette dernière, pour obtenir un couple de $100 \mathrm{mN}$ avec une machine longue $\left(k_{\mathrm{f}}=2\right)$, contre $800 \mathrm{~g}$ pour la machine vernier. La masse d'aimant diminue et les écarts s'estompent lorsque la machine se raccourci.

Un autre caractère important des moteurs est l'aptitude aux surcouples. Il est parfois nécessaire de pouvoir disposer transitoirement d'un couple largement supérieur au couple nominal en augmentant le courant d'alimentation. Pour la machine synchrone classique, il est facile d'obtenir des surcouples importants, surtout lorsque le nombre de pôles est élevé. En effet, un accroissement important du courant n'entraîne ici qu'un accroissement faible du champ dans l'entrefer, la machine n'a alors pas tendance à saturer et la contrainte de démagnétisation des aimants reste limitée. Il n'en va pas de même dans les machines vernier. Même si le problème de démagnétisation ne se pose pas non plus ici (les machines sont pour la plupart dimensionnées avec un facteur de sécurité au moins égal à 2 concernant le champ démagnétisant) ces machines fonctionnent avec un champ de réaction d'induit important qui oblige à largement surdimensionner les culasses de fermeture des flux pour permettre les surcouples. Les

\footnotetext{
$\left({ }^{4}\right)$ Les matériaux utilisés aujourd'hui seront vraisemblablement toujours chers, mais il est certain que les progrès réalisés dans ces domaines permettront la mise au point, tôt ou tard, d'aimants meilleur marché.
} 
performances massiques s'en trouvent affectées. On peut approximativement estimer à $20 \%$ l'augmentation de poids du moteur permettant un surcouple double du couple nominal (pour un moteur vernier à aimants dimensionné au couple nominal avec une marge de surcouple réduite).

\section{Conclusion}

Nous pouvons résumer, en guise de conclusion, les principales propriétés des machines présentées, dans le stricte cadre de cette comparaison. La structure classique est performante, en atteste son succès actuel, et semble être le meilleur choix dans la plupart des cas si l'on ne recherche pas les performances extrêmes. La machine à réluctance variable à aimants permet, au prix de quelques inconvénients, d'aller plus loin dans les performances et doit être considérée comme une alternative intéressante, permettant de résoudre des problèmes d'encombrement très complexes. Pour ne citer qu'un exemple, bien d'actualité, prenons le cas des moteurs pour véhicules électriques. Il peut être avantageux d'intégrer directement le moteur dans les roues, certains constructeurs font déjà des tentatives dans ce domaine, or les performances requises en encombrement et masse sont telles qu'aucune solution classique ne s'avère véritablement satisfaisante. Les caractéristiques du moteur vernier à aimants sont bien adaptées à cette application, la structure de cet actionneur est très simple, il est possible d'obtenir des couples très important dans le volume réduit d'une roue et la masse de l'ensemble (le couple massique peut être supérieur à $15 \mathrm{mN} / \mathrm{kg}$ ) ne serait pas prohibitive pour un véhicule à vocation urbaine.

\section{Bibliographie}

[1] Mukherji K.C. et Tustin A., Vernier Reluctance Motor, IEE, 9 (1974).

[2] Bastos J.P., Goyet R. et Lucidarme J., Performances intrinsèques des machines à réluctance variable à disque imbriqués, RPA, 15 (1980).

[3] Drouet G., Contribution à l'étude des machines à réluctance à commutation électronique excitées par courants statoriques, Thèse de Doctorat, INPT (Toulouse, mars 1984).

[4] Brissoneau P., Brugel L., Kiener A., Lebouc D. et Yonnet J.P., Nouvelles structures de moteurs à aimants permanents, RGE, 10 (1984).

[5] Andrieux C., Réalisation d'un ensemble à vitesse variable de dynamique élevée, Thèse de Doctorat, INPT (Toulouse, mars 1986).

[6] Lucidarme J., Rioux C. et Pouillange J.P., Moteurs discoïde à réluctance variable et à aimants permanents : des couples spécifiques élevés à faible vitesse, RGE, $n^{\circ} 3$ (mars 1987) pp. 48-52.

[7] Matt D., Etude de deux structures originales de machine à réluctance variable polyentrefer, Thèse de Doctorat, Université Paris 6 (avril 1987).

[8] Desesquelles P.F., Etude paramétrique des performances intrinsèques des machines multi-entrefer à réluctance et aimants alternés, Thèse de Doctorat d'Etat (Université Paris 6, avril 1988).

[9] Closa D., Conception, réalisation et essais de machines à aimants permanents et commutation électronique à encombrement axial réduit, Thèse de Doctorat, INP'T (Toulouse, septembre 1988).

[10] Matt D., Goyet R., Haddad N., Lucidarme J. et Rioux C., Study of Original Structures of Multiairgap Reluctance Machines, ICEM (Pise, 1988).

[11] Nogarede B., Etude de moteurs sans encoches à aimants permanents de forte pussance à basse vitesse, Thèse de Doctorat, INPT (Toulouse, juin 1990).

[12] Chillet C., Yonnet J.P. et Brissoneau P., Machines synchrones à aimants permanents : structures à entrefer plan, RGE, 6 (1990). 
[13] Desesquelles P.F., Lucidarme J. et Ben Ahmed A., Theorical and Experimental Results upon Multi-airgap Axial Synchronous Machines with Permanent Magnets, ICEM Cambridge Ma. (USA, août 1990).

[14] Weh H., May H. et Shalaby M., Higly Effective Magnetic Circuits for Permanent Magnet Excited Synchronous Machines, ICEM Cambridge Ma. (USA, août 1990).

[15] Ben Ahmed A., Desesquelles P.F. et Lucidarme J., Teeth Topology and Optimisation of Permanent Magnets Synchronous Machines, International Conference on the Evolution and Modern Aspect of Synchronous Machines (Août 1991).

[16] Gueraud A., Pouillange J.P. et Cremet G., Evolution des performances et nouvelles applications des machines à aimants permanents, RGE, 4 (1991).

[17] Poulot P., Matt D. et Regord C., Machines synchrones à aimants alternés et concentration de flux, lère Conférence J.C.G.E. (Toulouse, avril 1992).

[18] Llibre J.F. et Matt D., Vernier Reluctance Magnet Machine for Electric Vehicle, ICEM (Paris 1994). 